ISSN: 2638-5007

Volume 2, Issue 2, 2019, PP: 28-30

\title{
Type II Hereditary Angioedema Misdiagnosed with an Acute Abdomen: A Case Report Study
}

\author{
Daniela A. Haehn" ${ }^{1}$, Maritza G. Rivera-Valenzuela² ${ }^{2}$ Alexei Gonzalez-Estrada ${ }^{2 *}$ \\ ${ }^{1}$ Department of Anesthesia and Perioperative Medicine, Mayo Clinic Florida, Jacksonville, FL, U.S.A. \\ ${ }^{2}$ Division of Pulmonary, Allergy and Sleep Medicine, Department of Medicine, Mayo Clinic Florida, FL, U.S.A. \\ gonzalez.alexei@mayo.edu \\ *Corresponding Author: Alexei Gonzalez-Estrada, Assistant Professor, Division of Pulmonary, Allergy and Sleep \\ Medicine, Department of Medicine, Mayo Clinic Florida, Jacksonville, FL,USA.
}

\section{Abstract}

Hereditary angioedema (HAE) is a rare autosomal dominant condition caused by an uncontrolled activation of the contact pathway leading to an excessive production of bradykinin, with subsequent episodes of self-limited, non-pruritic swelling of the skin, the gastrointestinal and airway mucosa, which can be life-threatening. Here, we present a case of type II HAE in a patient who presented to multiple institutions with severe abdominal crisis described as diffuse colicky abdominal pain associated with abdominal distention and was misdiagnosed with acute abdomen leading to multiple surgical procedures.

Keywords: Abdominal attacks, type II Hereditary Angiodema, angioedema, acute abdomen

\section{INTRODUCTION}

C1- inhibitor hereditary angioedema (C1-INH-HAE) is an autosomal dominant condition caused by an excessive production of bradykinin due to a mutation in SERPING1 gene that encodes for C1-Inhibitor (C1-INH) protein, leading to an uncontrolled auto activation of contact activation pathway [1, 2]. HAE can be classified based on the C1-INH serum levels and its function. Patients with C1-INH deficiency are classified as Type I, while patients with Type II HAE have normal or even high levels of C1-INH with poor function [3]. A new type of HAE has been described where levels and function of $\mathrm{C} 1$ remains within the normal ranges. HAE with normal C1-INH can be associated with mutations in different genes such as Factor XII, angiopoietin 1 and plasminogen [4]. Symptoms of HAE include recurrent two to five days long episodes of self-limited, non-pruritic swelling of the skin, the gastrointestinal and airway mucosa, which can be life-threatening. Abdominal pain is a common manifestation of patients with HAE caused by a transient bowel obstruction due to mucosal edema. This clinical picture is often misdiagnosed as acute abdomen, sudden onset of severe abdominal pain, leading to unnecessary surgical procedures. Here, a case of a patient with type II HAE with gastrointestinal tract manifestations is presented.

\section{CASE REPORT}

A 59-year old female presented to the allergy clinic for evaluation of recurrent episodes of severe abdominal pain since childhood associated with swelling on her abdomen, neck and extremities which were triggered by stress and trauma (Figure $1 \mathrm{~A}-\mathrm{B}$ ). The edema of her skin was non-pruritic and without signs of urticaria. As per the patient, those attacks lasted up to two days resolving spontaneously without treatment. However, occasionally she presented with severe abdominal crisis described as diffuse colicky abdominal pain associated with abdominal distention. These episodes brought her to the emergency department leaving her with two exploratory laparotomies by the age of eighteen without revealing an etiology for her misdiagnosed surgical emergencies. Past medical history included antiphospholipid syndrome (APAS). Medications associated with acquired angioedema were ruled out (e.i., angiotensin-converting enzyme inhibitors, non-steroideal anti-inflammatory drugs, angiotensin-2 receptor blockers). Family history was 
significant for HAE type II in her mother. After a lifetime of multiple medical investigations from different providers she was diagnosed with HAE. Physical examination at the time of consult was noncontributory (Figure 2 A - B). Laboratory workup during one of her acute attacks showed low $\mathrm{C} 4$ of $<8 \mathrm{mg} / \mathrm{dl}$ (reference: $16-48 \mathrm{mg} / \mathrm{dl}$ ), C1-INH levels of $28 \mathrm{mg} / \mathrm{dL}$ (reference: 19-37 mg/dL), and C1-

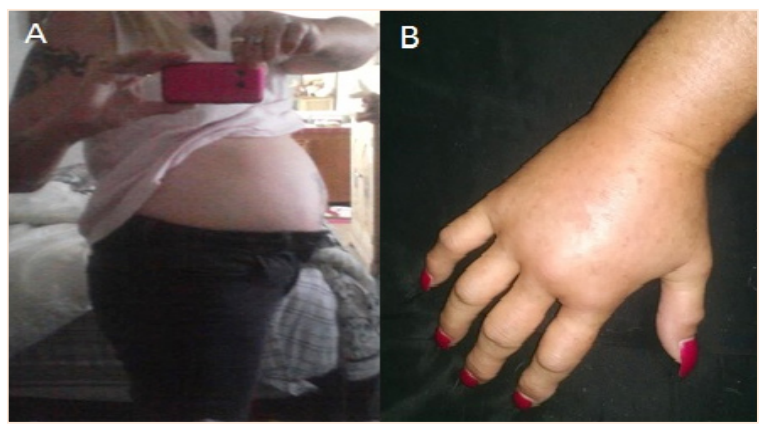

Fig 1
INH function levels of $3 \%$ with a repeat level of $6 \%$ one month later (reference: normal: $>67 \%$ normal; equivocal: $41-67 \%$ normal, Abnormal: $<41 \%$ normal), confirming the diagnosis of Type II C1-INH HAE. The patient was treated with long term plasma-derived C1INH without further attacks and had good response to on-demand icatibant a BK receptor antagonist, and aminocaproic acid.

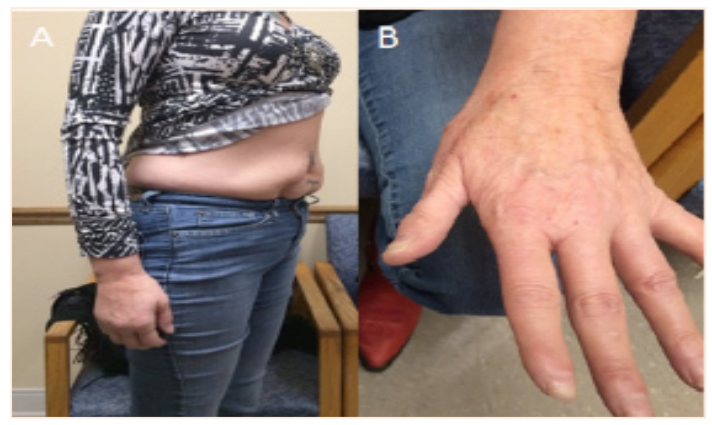

Fig 2

Fig 1. This is a figure showing the patient with severe angioedema of the abdomen during an abdominal attack of hereditary angioedema (A), subcutaneous edema of extremity (B).

Fig 2.The same patient normal abdomen (A) and extremity (B).

\section{DISCUSSION}

HAE is a rare condition with an estimated prevalence of 1 per 10,000 to 1 per 50,000 individuals[5] . It was first mentioned in 1876 when John Lawns Milton described HAE as "giant urticaria" and later was named "angioneurotic edema" by Quinke[6, 7]. Since then, the understanding of the pathophysiology of HAE and its diagnostic and treatment options have greatly evolved. However, given the rarity of this condition many of these patients remained misdiagnosed over the years.

C1-INH-HAE attacks may be triggered by stress, trauma, infections and/or medications. The frequency of attacks differs among patients and may vary throughout a patient's lifetime. The diagnosis of C1INH-HAE is based upon a suggestive clinical history and physical findings during episodes, combined with at least two sets of complement studies[3] . Treatment for C1-INH-HAE involves avoidance of triggers, prophylactic management to decrease the frequency of attacks, drug therapy and airway protection for acute episodes. Medication used for acute attacks include plasma derived C1-INH, recombinant human C1-INH concentrate, Bradykinin B2 Receptor antagonist, kallikrein inhibitors and solvent/detergent- treated plasma or fresh frozen plasma, and lanadelumab the more recently approved monoclonal antibody against kallikrein. Prophylactic treatment includes plasma derived C1-INH, and attenuated androgens.

Abdominal wall angioedema may be, although unusual, a subcutaneous/peripheral localization of HAE, it is not typical of acute abdomen nor is a common complaint in GI attacks of HAE, which are characterized by colicky pain, bloating distention, vomiting and diarrhea. This case illustrates the challenge of diagnosing HAE in patientswithgastrointestinal manifestations. Although the incidence of subcutaneous edema localized in the abdominal wall is well documented to be present in up to $93 \%$ of patients with HAE [8,9], those attacks are often misdiagnosed as acute abdomen leading to unnecessary surgical procedures, as in this case. Gastrointestinal attacks can occur independently or be associated with angioedema of the skin. Edema of the abdominal wall causes obstruction and distention that result in intense abdominal pain, which can be associated with other gastrointestinal symptoms such as nausea, vomiting, and diarrhea mimicking a surgical emergency. Additionally, during the acute episodes blood work-up may show leukocytosis with neutrophilia misleading the diagnosis [9]. Images may provide useful information, however they represent a challenge that depends on the period from the onset of symptoms and the radiologic evaluation [10]. Studies have reported that up to one third of patients with gastrointestinal manifestations undergo 
unnecessary laparotomies consequently leading to high patient burden $[9,11]$.

Data have shown that the final diagnosis of HAE is delayed by an average of 13 to 20 years after the first presentation of symptoms but may be sooner in some cases [5, 9]. Moreover, the delay is greater for type II C1INH-HAE than for the more common type I. Nowadays, guidelines have been issued in order to create a more standardized screening protocol for patients and their relatives. These initiatives might have a strong impact on economic burden since these patients undergo unnecessary medical procedures and multiple visits to different subspecialists before getting the correct diagnosis. Moreover, an early diagnosis can prevent life-threatening laryngeal attacks and improve the overall quality of life. All patients with suspected HAE due to C1-INH deficiency should be assessed for blood levels of serum C4, C1-INH protein, and C1-INH function[9].

\section{CONCLUSIONS}

Recurrent bouts of spasmodic, unexplained and self-limiting abdominal pain in those with a history of cutaneous or laryngeal angioedema should raise the suspicion of HAE. Abdominal pain is a common manifestation of C1-INH-HAE, and is often misdiagnosed as acute abdomen leading to unnecessary surgical procedures. Awareness of this presentation of HAE among primary providers is necessary to avoid economic and patient burden.

\section{REFERENCES}

[1] Wu, Y., Contact pathway of coagulation and inflammation. Thromb J, 2015. 13: p. 17.

[2] Cugno, M., et al., C1-inhibitor deficiency and angioedema: molecular mechanisms and clinical progress. Trends Mol Med, 2009. 15(2): p. 69-78.
[3] Cicardi, M., et al., Classification, diagnosis, and approach to treatment for angioedema: consensus report from the Hereditary Angioedema International Working Group. Allergy, 2014. 69(5): p. 602-16.

[4] Long, A.T., et al., Contact system revisited: an interface between inflammation, coagulation, and innate immunity. J Thromb Haemost, 2016. 14(3): p. 427-37.

[5] Roche, O., et al., Hereditary angioedema due to C1 inhibitor deficiency: patient registry and approach to the prevalence in Spain. Ann Allergy Asthma Immunol, 2005. 94(4): p. 498-503.

[6] Khan, D.A., Hereditary angioedema: Historical aspects, classification, pathophysiology, clinical presentation, and laboratory diagnosis. Allergy Asthma Proc, 2011. 32(1): p. 1-10.

[7] Umerani, M.S., K. Alzahrani, and G.A. Mostafa, Hereditary angioedema: A rare presentation after anterior cervical discectomy and fusion. Asian J Neurosurg, 2015. 10(3): p. 253-5.

[8] Bork, K., et al., Hereditary angioedema: new findings concerning symptoms, affected organs, and course. Am J Med, 2006. 119(3): p. 267-74.

[9] Henao, M.P., et al., Diagnosis and screening of patients with hereditary angioedema in primary care. Ther Clin Risk Manag, 2016. 12: p. 701-11.

[10] Gakhal, M.S. and G.V. Marcotte, Hereditary angioedema: imaging manifestations and clinical management. Emerg Radiol, 2015. 22(1): p. 83-90.

[11] Rubinstein, E., et al., Abdominal attacks and treatment in hereditary angioedema with C1inhibitor deficiency. BMC Gastroenterol, 2014. 14: p. 71.

Citation: Daniela A. Haehn, Maritza G. Rivera-Valenzuela, Alexei Gonzalez-Estrada. Type II Hereditary Angioedema Misdiagnosed with an Acute Abdomen: A Case Report Study. Archives of Emergency Medicine and Intensive Care. 2019; 2(2): 28-30.

Copyright: (C) 2019 Daniela A. Haehn, Maritza G. Rivera-Valenzuela, Alexei Gonzalez-Estrada. This is an open access article distributed under the Creative Commons Attribution License, which permits unrestricted use, distribution, and reproduction in any medium, provided the original work is properly cited. 